\title{
Enhancement of Speech Signal Corrupted by Impulsive Noise Using Rank Order Mean
}

\author{
Arun Kumar Singh ${ }^{1}$, Prof. Neelam Srivastava ${ }^{2}$, Er. Piyush Singh ${ }^{3}$ \\ ${ }^{1,2,3}$ Department of Electronics Engineering, Institute of Engineering and Technology, Lucknow, India
}

\begin{abstract}
Speech is the medium through which information can be conveyed from one person to another. Audible frequency range of human beings is between $20 \mathrm{~Hz}$ to $20 \mathrm{kHz}$, significant frequency components has range up to $4 \mathrm{kHz}$. Rank Order Mean method has been proposed in this work which is used for ejection of impulse noise from the speech signal using Rank. To estimate the time occurrence of impulsive noise rank order differentiation is applied at the input signal. Then rank order mean is used for replacing the noisy samples to get the noise free signal. The above mentioned technique shows betterment in results in terms of Signal to Noise Ratio (SNR) and Log Spectral Distance (LSD) when compare to the discrete wavelet transform and adaptive median filtering techniques.Simulation work have been done on the Matlab software.
\end{abstract}

Keywords: Speech enhancement,impulse noise,mean filter,signal to noise ratio(SNR)

\section{Introduction}

When the information is transmitted from source to receiver, noise from the surroundings gets added to the signal. The resultant signal content two components, one carries the information of interest means the useful signal, the other error generated is because of superimposition with the original signal. Speech is the most important form of human communication. For that reason, there is a big trend to increase the quality of speech signal and improve tele communications limitations. The random error and noise are unwanted because they degrade the accuracy and precision of the measured signal. There are different noises which may affect the signal under consideration,these noises are White noise,Colored noise,Brown noise,Pink noise and Impulse noise. White noise is a sound or signal consisting of all audible frequencies with equal intensity. Any noise that is not white can be termed as colored noise. Colored noise has frequency spectrum that is limited within a range unlike white noise which extends over the entire spectrum.There are different types of colored noise (brown noise, pink noise, etc.) depending upon the gradation in the Power Spectral Density (PSD) of the noise. Colored noise can be generated by passing white noise through a filter with required frequency response.

Impulsive noise refers to sudden bursts of noise with relatively high amplitude. This type of noise causes click sounds in the signal of interest. Impulsive noise is generally modelled as contaminated Gaussian noise [4].The ratio of the variances of the two Gaussian noises decides the impulsive Character of the noise generated. Impulsive noise corrupt thousands of sample. There are number of methods to overcome this problem. Some of them are methods based on: wavelets [6],soft decision and recursion, diffusion filtering[11] Baysian frameworks[5].But above mentioned techniques are not so much efficient. The proposed technique works in following steps:

- First detecting the noisy samples using the technique rank order difference by setting a threshold.

- In this step detected noisy samples will be replaced by clean samples using the technique rank order mean.

\section{Detection and Cleaning Algorithm}

To accomplish the task of detection and cleaning following steps are followed.

- Taking the sliding window of size five as shown in the figure.

\begin{tabular}{|l|l|l|l|l|}
\hline $\mathrm{x}(\mathrm{n}-2)$ & $\mathrm{x}(\mathrm{n}-1)$ & $\mathrm{x}(\mathrm{n})$ & $\mathrm{x}(\mathrm{n}+1)$ & $\mathrm{x}(\mathrm{n}+2)$ \\
\hline
\end{tabular}

- Consider a vector $\mathrm{x}(\mathrm{n})$ of size five centered at $\mathrm{n}$.

- Define a vector of size four that excudes $\mathrm{x}(\mathrm{n})$, the centre sample under inspection

$$
\begin{aligned}
& w(n)=\left[w_{1}(n), w_{2}(n), w_{3}(n), w_{4}(n)\right] \\
& \quad=[x(n-2), x(n-1), x(n+1), x(n+2)]
\end{aligned}
$$

- The observation samples from $\mathrm{w}(\mathrm{n})$ are sorted,

$$
r(n)=\left[r_{1}(n), r_{2}(n), r_{3}(n), r_{4}(n)\right]
$$

where the elements in $r(n)$ are ordered by rank i.e

$$
r_{1}(n) \leq r_{2}(n) \leq r_{3}(n) \leq r_{4}(n)
$$

- rank order difference defind as

$$
d_{i}(n)=\left\{\begin{array}{cc}
r_{i}(n)-x(n), & \text { if } x(n) \leq \mu(n) \\
x(n)-r_{4-i}(n), & \text { if } x(n)>\mu(n)
\end{array}\right.
$$

Where

$$
\mu(n)=\left[r_{2}(n)+r_{3}(n)\right] / 2
$$

is called the rank order mean(ROM).For a window of size five, $i=1,2$.

- The algorithm decides $\mathrm{x}(\mathrm{n})$ is a noise if any of the following conditions hold

$$
d_{i}>T_{i} \text { for } i=1,2
$$

- Where T1 and T2 are two appropriately chosen threshold - Every detected impulse is replaced by the ROM, $\mu(\mathrm{n})$. 


\section{Methodology}

- Signal is segmented using the window of size 5.

- Then Rank Order Differentiation is applied.

- To determine the impulse noise location in the signal rank order differentiation is applied.

- The noisy samples are replaced by rank order mean.

\section{Implementation Results}

\section{A. Speech data base}

In the test data set, the voice was recorded with a Compaq iPAQ 3630 built-in microphone and an Optimus Nova 80 close-talk microphone. The iPAQ data were recorded using the $\mathrm{AD}$ converter in the $\mathrm{iPAQ}$, and the close-talk data were recorded using a Creative Sound board. Both channels were recorded with a $11.025 \mathrm{kHz}$ sampling.

\section{B. Impulse noise modeling}

The noise is modeled in 2 stages. In the first stage of noise modeling process,2state Markovian state transition is used[12].One state consider as impulsive and the other state consider as non impulsive state.In the Figure 1 state B is considered as impulsive state i.e this stage is contaminated with noise.State A is considered as non impulsive state i.e noise process is considered to be switched off in this state.

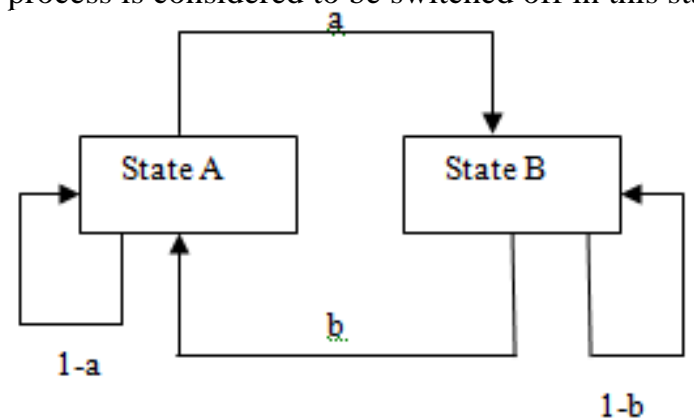

Figure 1: 2 State Markovian process[12]

Second stage of modeling completes with the multiplication of Gaussian distribution having zero mean with the data stream generated in the above stage.the result will be the very short occuring Gaussian distributed noise.Following waveforms are given using Matlab to accomplish the task.

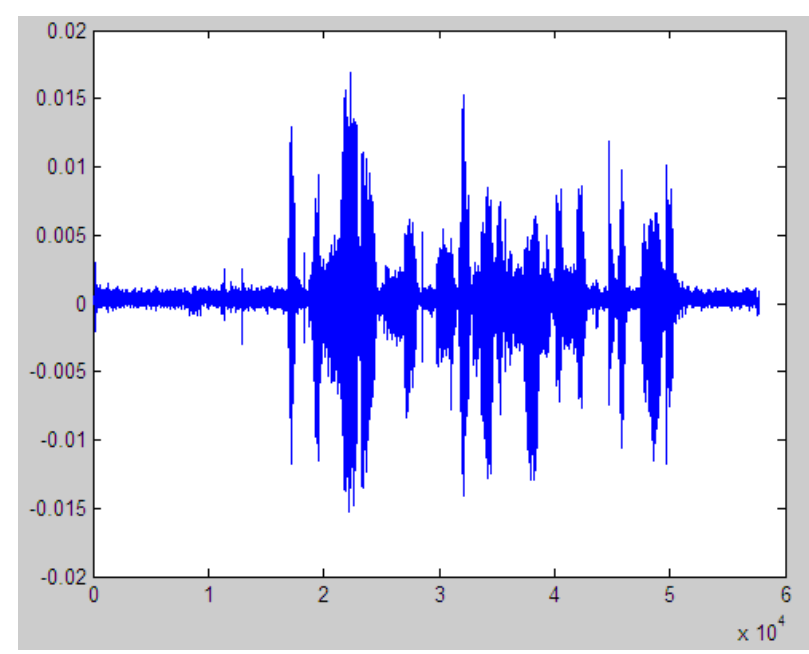

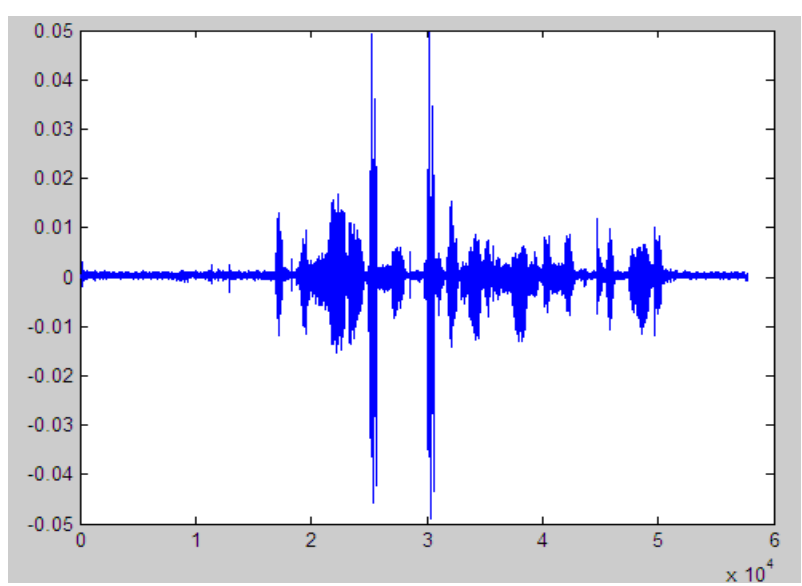

Figure 2: The first plot shows a given number of samples of a speech signal which is uncorrupted by the impulsive noise. The second plot shows the speech signal of given number of samples corrupted by the impulsive noise at a certain range of samples with the higher magnitude compare to the uncorrupted signal.

\section{Signal to noise ratio and Log Spectral Distance}

The above described techniq shows improvement in terms of Signal to Noise Ratio (SNR) and Log spectral distance(LSD). All the Simulations are done using matlab.
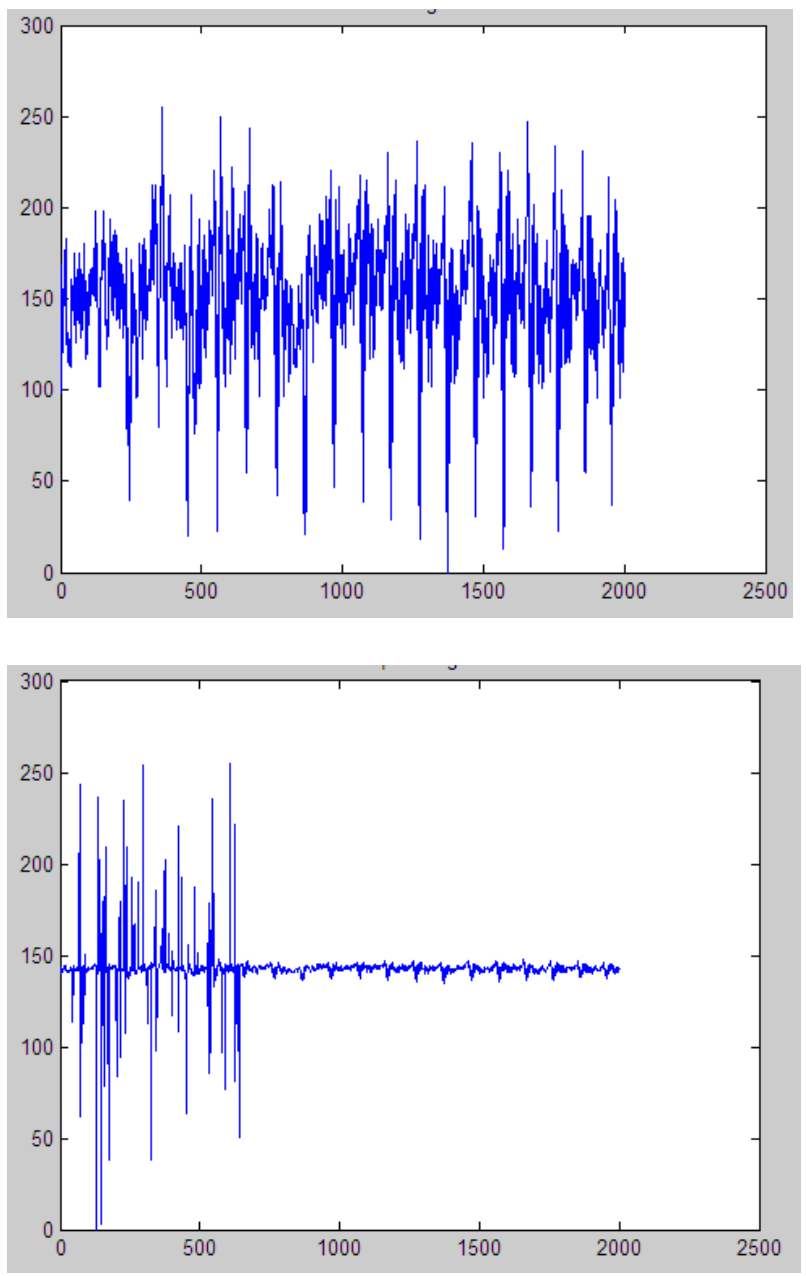


\section{International Journal of Science and Research (IJSR) \\ ISSN (Online): 2319-7064}

Index Copernicus Value (2013): 6.14 | Impact Factor (2015): 6.391

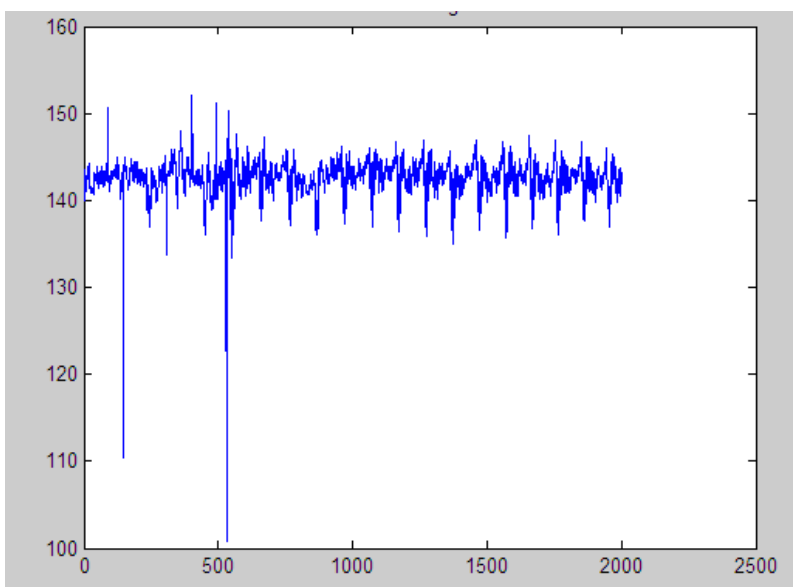

Figure 3: The first shows a pure speech signal at a sampling frequence of $11.025 \mathrm{kHz}$. The second plot shows the speech signal corrupted by the impulsive noise. The third plot shows the output of the proposed technique.It can be sen that impulsive noise removed effectively.

Table 1: The improvement in the SNR and LSD for proposed technique when compare to the discrete wavelet transform and Adaptive Median filtering technique

\begin{tabular}{|c|c|c|}
\hline Performance Parameter & $\begin{array}{c}\text { Proposed } \\
\text { technique }\end{array}$ & $\begin{array}{c}\text { Adaptive Median } \\
\text { filtering }\end{array}$ \\
\hline Signal to noise ratio(SNR) & 11.4921 & -7.3164 \\
\hline Log spectral distance (LSD) & 2.3397 & 1.5996 \\
\hline
\end{tabular}

\section{Conclusion}

Speech enhancement is a special case of signal estimation as speech is non stationary and hence human ear is the final judge and it requires a mathematical error criterion. To evaluate their speech enhancement capabilities, all the parameters are performed on computer simulations. The results show that Rank Order Mean method gave the better noise reduction capability in comparison to given speech enhancement method. Future work might involve a real time implementation of the system so that it could be used in devices where speech quality and speech intelligibility is must.

\section{References}

[1] Swamy, Prateek Basavapur, Rohini S. Hallikar, and M. Uttara Kumari. "Enhancement of speech signals corrupted by impulsive noise using wavelets and adaptive median filtering." In Signal Processing, Computing and Control (ISPCC), 2013 IEEE International Conference on, pp. 1-6. IEEE, 2013.

[2] Renjie Tong, Yingyue Zhou, Long Zhang, Guangzhao Bao, and Zhongfu Ye, "A Robust Time-Frequency Decomposition Model for Suppression of Mixed Gaussian-Impulse Noise in Audio Signals ",IEEE/ACM Transactions on Audio, Speech, and Language Processing, Vol. 23, No. 1, January 2015.

[3] Avila F., Biscainho L., "Bayesian Restoration of Audio Signals Degraded by Impulsive Noise Modeled as Individual Pulses", IEEE Transactions On Audio, Speech, And Language Processing, 20, 9, 2470-2481.
[4] Marco Ruhland, Joerg Bitzer, "Reduction of Gaussian, Supergaussian, and Impulsive Noise by Interpolation of the Binary Mask Residual", IEEE/ACM Transaction on Audio, Speech, and Language Processing, Vol. 23, No. 10, October 2015.

[5] J. Murphy and S. Godsill, "Joint Bayesian removal of impulse andbackground noise," in Proceedings of IEEE International Conference on Acoustics, Speech and Signal Processing (ICASSP), pp. 261-264, IEEE, 2011.

[6] R. C. Nongpiur, "Impulse noise removal in speech using wavelets," in Acoustics, Speech and Signal Processing, 2008. ICASSP 2008. IEEE International Conference on. IEEE, 2008, pp. 1593-1596

[7] A. Subramanya, M. L. Seltzer, and A. Acero, "Automatic removal of typed keystrokes from speech signals," Signal Processing Letters, IEEE,vol. 14, no. 5, pp. 363-366, 2007.

[8] C. Chandra, M. S. Moore, and S. K. Mitra, "An efficient method for the removal of impulse noise from speech and audio signals," in Circuits and Systems, 1998. ISCAS'98. Proceedings of the 1998 IEEEInternational Symposium on, vol. 4. IEEE, 1998, pp. 206-208.

[9] Z. He, X. Guo, and M. Zhang, "Detection and removal of impulsive colored noise for speech enhancement," in Information and Automation(ICIA), 2010 IEEE International Conference on. IEEE, 2010, pp. 23202324.

[10] S. Zahedpour, S. Feizi, A. Amini, M. Ferdosizadeh, and F. Marvasti, "Impulsive noise cancellation based on soft decision and recursion," Instrumentation and Measurement, IEEE Transactions on, vol. 58, no. 8, pp. 2780-2790, 2009.

[11] R. Talmon, I. Cohen, and S. Gannot, "Speech enhancement in transient noise environment using diffusion filtering," in Acoustics Speech and Signal Processing (ICASSP), 2010 IEEE International Conference on. IEEE, 2010, pp. 4782-4785.

[12] S. V. Vaseghi, "Advanced digital signal processing and noise reduction." Wiley, 2008, pp. 362-364.

[13] A. Graps, "An introduction to wavelets," Computational Science \& Engineering, IEEE, vol. 2, no. 2, pp. 50-61, 1995

[14] Paliwal, K.K, Atal, B.S. Efficient vector quantization of LPC Parameters at 24 bits / frame. IEEE Trans. Speech Audio Process, pp.3- 14, 1993.

[15] J.G. Proakis and D.G. Manolakis, "Digital Signal Processing: Principles, Algorithms and Applications," Third ed., Prentice Hall, NJ, 1996. 